\title{
Coeficiente de cultura da alface hidropônica baseado no conceito de graus-dia
}

\author{
Carlos Roberto Weide Moura ${ }^{1}$, Sérgio Zolnier ${ }^{2}$, Aristides Ribeiro ${ }^{3}$, Rubens Alves de Oliveira ${ }^{4}$
}

\begin{abstract}
RESUMO
O objetivo principal deste trabalho foi propor a adoção de um coeficiente de cultura térmico, com a aplicação do conceito de graus-dia acumulados após o transplantio, para estimativa da evapotranspiração diária (ET) dos cultivares de alface Grand Rapids, Regina e Great Lakes em sistema hidropônico do tipo NFT (“nutrient film technique”), em ambiente protegido. Posteriormente, o modelo matemático ajustado aos valores observados de $\mathrm{K}_{\mathrm{c}}$ (coeficiente de cultura) foi utilizado para a estimativa de ET no período pós-transplantio por meio dos métodos: Penman-Monteith parametrizado pela FAO, em 1998 (PMF); Penman-Monteith modificado para condições aerodinâmicas, características de ambientes protegidos (PMAP); Penman-Monteith para ambiente protegido simplificado (PMAPS); Priestley-Taylor (PT); Radiação solar (RS); e Radiação solar simplificado (RSS). Verificou-se que o modelo sigmoidal com quatro parâmetros ajustou-se bem aos valores observados de $\mathrm{K}_{c}$, proporcionando coeficientes de determinação ajustados de 0,99, 0,98 e 0,98 para os cultivares Grand Rapids, Regina e Great Lakes, respectivamente. No que se refere às estimativas diárias de $\mathrm{ET}_{c}$, os métodos PMF, PMAP e RS apresentaram melhor desempenho estatístico em relação aos métodos PMAPS, PT e RSS, independentemente do cultivar avaliado. No entanto, o método PMF subestimou sistematicamente a ET ${ }_{c}$ no período inicial de crescimento da alface.
\end{abstract}

Palavras chave: Cultivo protegido, tempo térmico, técnica de circulação laminar de nutrientes.

\section{ABSTRACT}

\section{Degree-day-based crop coefficient for hydroponic lettuce}

The main objective in this work was to propose the adoption of a thermal crop coefficient by using accumulated degree-days after transplanting to estimate lettuce daily evapotranspiration (ET) for the cultivars Grand Rapids, Regina and Great Lakes in a NFT hydroponic system under protected environment. Subsequently, the adjusted model to the observed values of $\mathrm{K}_{c}$ was used for estimating $\mathrm{ET}_{c}$ during the growing period after transplanting with the following methods: Penman-Monteith parameterized by FAO in 1998 (PMF); Penman-Monteith modified for aerodynamic characteristics in protected environment (PMAP); simplified Penman-Monteith (PMAPS); Priestley-Taylor (PT); solar radiation (RS); and simplified solar radiation (RSS). It was verified that the sigmoid model with four parameters fitted well to observed values of $\mathrm{K}_{\mathrm{c}}$,giving adjusted coefficients of determination of 0,9874, 0,9784 and 0,9767 for cultivars Grand Rapids, Regina and Great Lakes, respectively. As far as daily $\mathrm{ET}_{c}$ estimates are concerned, the methods PMF, PMAP and RS presented better statistical performance as compared to the methods PMAPS, PT and RSS, regardless of the evaluated cultivar. However, the method PMF systematically underestimated $\mathrm{ET}_{\mathrm{c}}$ in the lettuce initial growth period.

Key words: Protected cultivation, thermal time, nutrient film technique.

Recebido para publicação em março de 2008 e aprovado em dezembro de 2009

${ }^{1}$ Meteorologista, Mestre. Centro de Previsão de Tempo e Estudos Climáticos - CPTEC, INPE, Av. dos Astronautas, 1758, Jd. Granja, 12227-010, São José dos Campos, São Paulo, Brasil. carloswmoura@hotmail.com

${ }^{2}$ Engenheiro Agrícola, Ph.D. Departamento de Engenharia Agrícola (DEA), Universidade Federal de Viçosa, Av. P. H. Holfs, s/n, 36570-000, Viçosa, Minas Gerais (MG), Brasil. zolnier@ufv.br

${ }^{3}$ Engenheiro-Agrônomo, Doutor. DEA, Universidade Federal de Viçosa, Av. P. H. Holfs, s/n, 36570-000, Viçosa, MG, Brasil. ribeiro@ufv.br

${ }^{4}$ Engenheiro Agrícola, Doutor. DEA, Universidade Federal de Viçosa, Av. P. H. Holfs, s/n, 36570-000, Viçosa, MG, Brasil. rubens@ufv.br 


\section{INTRODUÇÃO}

O cultivo de alface (Lactuca sativa L.) em sistemas hidropônicos do tipo NFT (“nutrient film technique”) em ambiente protegido tem grande potencial para aprimorar o uso eficiente de água e de fertilizantes, bem como minimizar problemas ambientais decorrentes da contaminação do lençol freático pela lixiviação de componentes químicos. No entanto, para avaliação do uso eficiente de água nesses sistemas ao longo do ciclo da cultura, em comparação ao cultivo em condições de campo, são necessárias medições ou estimativas da taxa diária de evapotranspiração (ET $_{c}$ e de crescimento da cultura.

Em condições de campo, a ET diária pode ser estimada por meio da multiplicação do coeficiente de cultura $\left(\mathrm{K}_{\mathrm{c}}\right)$ pela evapotranspiração potencial $\left(\mathrm{ET}_{\mathrm{p}}\right)$, adotando-se um método específico, conforme a disponibilidade de dados meteorológicos (Doorenbos \& Pruitt, 1977). Com a implantação de redes automáticas de superfície em escala global para medição das variáveis meteorológicas, a ET foi substituída pela evapotranspiração de referência ( $\left.\mathrm{ET}_{0}\right)^{\mathrm{p}}$, conforme a parametrização do modelo de PenmanMonteith proposta por Allen et al. (1998). Infelizmente, os dados medidos nas estações meteorológicas automáticas não podem ser utilizados diretamente para estimativa do uso de água em ambiente protegido, em razão de características microclimáticas específicas desse sistema de cultivo, as quais são influenciadas, principalmente, pela transmissibilidade do material de cobertura e taxa de ventilação da instalação (Hanan, 1998). Dessa forma, a utilização da metodologia sugerida por Doorenbos \& Pruitt (1977), para estimativa da $\mathrm{ET}_{\mathrm{c}}$ da alface cultivada em sistema hidropônico em condições de ambiente protegido, está condicionada à estimativa do $\mathrm{K}_{\mathrm{c}}$ após o transplantio, assim como à estimativa da evapotranspiração potencial ou de referência com base em medições das variáveis ambientais no interior da instalação.

O desenvolvimento e a validação de modelos matemáticos, que possibilitam estimativas do coeficiente de cultura em sistemas de cultivos específicos, têm sido realizados por diversos pesquisadores. Amayreh \& AlAbed (2005) determinaram o coeficiente de cultura do tomateiro em condições de campo a partir da utilização de dados meteorológicos e de medições de ET de plantas cultivadas em solo coberto com filmes plásticos pretos e irrigadas por gotejamento. Os autores observaram que os valores de $\mathrm{K}_{\mathrm{c}}$ obtidos em meados do ciclo de crescimento, nessas condições específicas de cultivo, foram 31\% menores que os recomendados por Doorenbos \& Pruitt (1977).

Jayanthi et al. (2007) propuseram o uso de medições de reflexão do dossel vegetativo para quantificar a expansão foliar e proporcionar estimativas do coeficiente de cultura da batata no Estado de Idaho, situado nos Estados Unidos. Similarmente, o tempo térmico pode ser adotado para estimativa do índice de área foliar e, consequentemente, do coeficiente de cultura. Orgaz et al. (2005) utilizaram o conceito de graus-dia acumulados para ajustar modelos de estimativa do $\mathrm{K}_{\mathrm{c}}$ ao longo do ciclo de crescimento das culturas do melão, feijão, pimentão e melancia. O tempo térmico acumulado também foi utilizado por Oliveira et al. (2005) para estimativa do coeficiente de cultura da alface com formação de cabeça, a qual foi cultivada em solo e irrigada por gotejamento subsuperficial.

No presente trabalho teve-se por objetivo principal propor a adoção de um coeficiente de cultura térmico, com a aplicação do conceito de graus-dia acumulados após o transplantio, para a estimativa da evapotranspiração diária da alface cultivada em sistema hidropônico do tipo NFT em ambiente protegido. Posteriormente, os valores de $\mathrm{K}_{\mathrm{c}}$ estimados no período pós-transplantio foram utilizados em vários modelos de estimativa de $\mathrm{ET}_{\mathrm{c}}$, incluindo métodos baseados em princípios físicos, como no modelo de Penman-Monteith, assim como em métodos empíricos.

\section{MATERIAL E MÉTODOS}

\section{Local do experimento e medição das variáveis meteorológicas e do consumo de água}

As medições das variáveis meteorológicas no ambiente de cultivo e do consumo de água da alface foram realizadas em dois experimentos independentes, conduzidos no setor de Meteorologia Agrícola, pertencente ao Departamento de Engenharia Agrícola (DEA), no campus da Universidade Federal de Viçosa (UFV), Viçosa, Minas Gerais. As coordenadas geográficas do local de execução do experimento são: latitude $20^{\circ} 45^{\prime}$ S, longitude $42^{\circ} 51^{\prime} \mathrm{O}$ e altitude $690 \mathrm{~m}$.

O consumo de água foi obtido utilizando-se os cultivares de alface Grand Rapids, Regina e Great Lakes, a partir do desenvolvimento de um sistema de medição específico para o cultivo hidropônico, o qual estava interligado ao sistema hidráulico das bancadas de cultivo (Zolnier et al., 2004). O desenvolvimento desse sistema permitiu a medição individual da $\mathrm{ET}_{\mathrm{c}}$ para cada unidade experimental, consistindo de 120 plantas, totalizando seis bancadas para os três cultivares avaliados. As medições de $\mathrm{ET}_{\mathrm{c}}$ foram realizadas ao longo do ciclo da cultura nos dois experimentos mencionados, sendo um conjunto de dados utilizado para determinação dos parâmetros dos métodos de estimativa da evapotranspiração, bem como do coeficiente de cultura, e o segundo conjunto foi aplicado para validação da $\mathrm{ET}_{\mathrm{c}}$ da alface a partir da multiplicação do $K_{c}$ térmico estimado pela $\mathrm{ET}_{\mathrm{p}}$ ou $\mathrm{ET}_{\mathrm{o}}$. Neste trabalho, o símbolo $\mathrm{ET}_{\mathrm{o}}$ refere-se à estimativa da 
evapotranspiração de uma cultura hipotética com resistência de superfície $\left(\mathrm{r}_{\mathrm{s}}\right)$ igual a $70 \mathrm{sm}^{-1}$, conforme sugerido por Allen et al. (1998).

Para determinação dos parâmetros dos métodos de estimativa da $\mathrm{ET}_{\mathrm{c}}$ e do $\mathrm{K}_{\mathrm{c}}$ da alface foram realizadas sete campanhas de medição, totalizando 21 dias úteis entre o transplantio e a colheita. No entanto, para validação das estimativas de $\mathrm{ET}_{\mathrm{c}}$ pelos diferentes métodos, foram utilizados valores de $\mathrm{ET}_{\mathrm{c}}$ obtidos em 15 dias úteis no período pós-transplantio, provenientes de cinco campanhas de medição. Nos dois experimentos, cada conjunto de dados foi constituído por três dias de medição de $\mathrm{ET}_{\mathrm{c}}$, seguidos por um intervalo de dois dias sem medições.

\section{Métodos de Estimativa da Evapotranspiração de Referência ou Potencial}

Seis métodos de estimativa de evapotranspiração da alface, cultivada em sistema hidropônico sob ambiente protegido, foram avaliados no presente estudo. Três deles são baseados em princípios físicos, tendo como referência o modelo de Penman-Monteith, com variações na parametrização da estimativa da resistência aerodinâmica e no número de variáveis meteorológicas exigidas para o cálculo da $\mathrm{ET}_{\mathrm{c}}$. Além disso, foram utilizados os métodos empíricos propostos por Priestley-Taylor, o método da radiação recomendado pela FAO e o método da radiação simplificado por Hargreaves \& Samani (1982). Breve descrição sobre as especificidades metodológicas será apresentada a seguir, sendo que as equações, as simbologias dos parâmetros e das variáveis associadas e as parametrizações adotadas estão apresentadas nas Tabelas 1 e 2 .

\section{Penman-Monteith-FAO (PMF)}

Este método é utilizado como referência para estimativa da evapotranspiração de uma cultura hipotética, a qual é posteriormente multiplicada pelo coeficiente de cultura $\left(\mathrm{K}_{\mathrm{c}}\right)$ para estimativa do uso de água pelas diferentes espécies vegetais ao longo do período de crescimento. Em sistemas hidropônicos, o fluxo de calor sensível corresponde ao aquecimento ou resfriamento da solução nutritiva (Zolnier et al., 2004). Para o cálculo dos parâmetros necessários para aplicação do método, foram utilizados os procedimentos propostos por Allen et al. (1998).

\section{Penman-Monteith Modificado para Ambientes Protegidos (PMAP)}

Neste método, a resistência ao fluxo de calor sensível da equação de Penman-Monteith foi determinada a partir da utilização do fator de desacoplamento $(\Omega)$, proposto por McNaughton \& Jarvis (1983). Esse fator é obtido pela relação $\left.1 /\left\{1+[\gamma /(\Delta+\gamma)]\left[r_{s} / r_{a}\right)\right]\right\}$, sendo os parâmetros descritos na Tabela 2. De acordo com os autores, o $\Omega$ médio para uma superfície de grama com crescimento rasteiro, cobrindo totalmente o solo, bem irrigada, e com albedo de 0,23 será aproximadamente 0,80 . Substituindo-se o valor $\Omega=$ 0,80 sugerido pelos autores e $\mathrm{r}_{\mathrm{s}}=70 \mathrm{~s} \mathrm{~m}^{-1}$, proposto por Allen et al. (1998), na relação usada para obtenção do fator de desacoplamento, a resistência aerodinâmica $\left(\mathrm{r}_{\mathrm{a}}\right)$ poderá ser estimada pela equação apresentada na Tabela 1.

A utilização do fator $\Omega$ para estimativa de $\mathrm{r}_{\mathrm{a}}$, em oposição à metodologia proposta por Allen et al. (1998), impede que os valores calculados desse parâmetro tendam para o infinito quando a velocidade do ar tender para zero

Tabela 1. Parametrização e equações correspondentes para estimativa da evapotranspiração de referência (LE $_{\mathrm{o}}$ ) e potencial (LE $)$ em ambiente protegido por meio da utilização dos métodos de Penman-Monteith-FAO (PMF), Penman-Monteith modificado para ambientes protegidos (PMAP), PMAP simplificado (PMAPS), Priestley-Taylor (PT), Radiação Solar (RS) e Radiação Solar Simplificado (RSS)

\begin{tabular}{|c|c|c|c|c|}
\hline Método & Dados do Ambiente & $r_{s}\left(s^{-1}\right)$ & $\mathbf{r}_{\mathrm{h}}\left(\mathrm{s} \mathrm{m}^{-1}\right)$ & Equação \\
\hline PMF & $\mathrm{R}_{\mathrm{g}}, \mathrm{t}, \mathrm{UR}, \mathrm{U}_{2}$ & 70 & $208 / U_{2}$ & $\mathrm{LE}_{\mathrm{o}}=\frac{\Delta\left(\mathrm{R}_{\mathrm{n}}-\mathrm{F}\right)+\rho_{\mathrm{ar}} \mathrm{c}_{\mathrm{par}} \mathrm{DPV} \mathrm{ar}_{\mathrm{ar}} / \mathrm{r}_{\mathrm{a}}}{\Delta+\gamma\left(1+\mathrm{r}_{\mathrm{s}} / \mathrm{r}_{\mathrm{a}}\right)}$ \\
\hline PMAP & $\mathrm{R}_{\mathrm{g}}, \mathrm{t}, \mathrm{UR}$ & 70 & $\mathrm{r}_{\mathrm{a}}=\frac{280 \gamma}{\gamma+\Delta}$ & $\mathrm{LE}_{\mathrm{o}}=\frac{\Delta\left(\mathrm{R}_{\mathrm{n}}-\mathrm{F}\right)+\rho_{\mathrm{ar}} \mathrm{c}_{\mathrm{par}} \mathrm{DPV}_{\mathrm{ar}} / \mathrm{r}_{\mathrm{a}}}{\Delta+\gamma\left(1+\mathrm{r}_{\mathrm{s}} / \mathrm{r}_{\mathrm{a}}\right)}$ \\
\hline PMAPS & $\mathrm{R}_{\mathrm{g}}, \mathrm{t}$ & 70 & $\mathrm{r}_{\mathrm{a}}=\frac{280 \gamma}{\gamma+\Delta}$ & $\mathrm{LE}_{\mathrm{o}}=\frac{\Delta\left(\mathrm{R}_{\mathrm{n}}-\mathrm{F}\right)+\rho_{\mathrm{ar}} \mathrm{c}_{\mathrm{par}}\left(\mathrm{e}_{\mathrm{s}}-\mathrm{e}_{\mathrm{s}_{-} \min }\right) / \mathrm{r}_{\mathrm{a}}}{\Delta+\gamma\left(1+\mathrm{r}_{\mathrm{s}} / \mathrm{r}_{\mathrm{a}}\right)}$ \\
\hline PT & $\mathrm{R}_{\mathrm{g}}, \mathrm{t}$ & - & - & $\mathrm{LE}_{\mathrm{p}}=\alpha \frac{\Delta}{\Delta+\gamma}\left(\mathrm{R}_{\mathrm{n}}-\mathrm{F}\right)$ \\
\hline RS & $\mathrm{R}_{\mathrm{g}}, \mathrm{t}$ & - & - & $\mathrm{LE}_{\mathrm{p}}=\mathrm{r} \frac{\Delta}{\Delta+\gamma} \mathrm{R}_{\mathrm{g}}$ \\
\hline RSS & $\mathrm{t}$ & - & - & $\mathrm{LE}_{\mathrm{p}}=\mathrm{r} \frac{\Delta}{\Delta+\gamma} \mathrm{k}_{\mathrm{r}} \sqrt{\mathrm{t}_{\mathrm{m}}-\mathrm{t}_{\min }} \mathrm{R}_{\mathrm{a}}$ \\
\hline
\end{tabular}


Tabela 2. Descrição dos símbolos adotados para representação das variáveis meteorológicas e dos parâmetros pertinentes aos métodos de estimativa da evapotranspiração de referência e potencial apresentados na Tabela 1

\begin{tabular}{|c|c|}
\hline Símbolo & Descrição \\
\hline $\mathrm{LE}_{\mathrm{o}}$ & fluxo de calor latente de referência à superfície da cultura hipotética $\left(\mathrm{MJ} \mathrm{d}^{-1} \mathrm{~m}^{-2}\right)$ \\
\hline $\mathrm{R}_{\mathrm{n}}$ & saldo de radiação à superfície $\left(\mathrm{MJ} \mathrm{d}^{-1} \mathrm{~m}^{-2}\right)$ \\
\hline $\mathrm{F}$ & fluxo de calor sensível na solução nutritiva, normalizado para a área da bancada de cultivo ( $\mathrm{MJ} \mathrm{d}^{-1} \mathrm{~m}^{-2}$ ) \\
\hline$\Delta$ & derivada da curva de pressão de saturação do vapor d’água com respeito à temperatura $\left(\mathrm{Pa}^{\circ} \mathrm{C}^{-1}\right)$ \\
\hline$\rho_{\mathrm{ar}}$ & densidade absoluta do ar $\left(\mathrm{kg} \mathrm{m}^{-3}\right)$ \\
\hline $\mathrm{C}_{\mathrm{par}}$ & calor específico do ar à pressão constante $\left(\mathrm{Jkg}^{-1}{ }^{\circ} \mathrm{C}^{-1}\right)$ \\
\hline $\mathrm{DPV}_{\mathrm{ar}}$ & déficit da pressão de saturação do vapor d’água no ar (Pa) \\
\hline$\gamma$ & coeficiente psicrométrico $\left(\mathrm{Pa}{ }^{\circ} \mathrm{C}^{-1}\right)$ \\
\hline$r_{a}$ & resistência ao fluxo de calor sensível $\left(\mathrm{s} \mathrm{m}^{-1}\right)$ \\
\hline$r_{s}$ & resistência de superfície da cultura de referência $\left(\mathrm{s} \mathrm{m}^{-1}\right)$ \\
\hline$\Omega$ & fator de desacoplamento (adimensional) \\
\hline $\mathrm{e}_{\mathrm{s}}$ & pressão de saturação de vapor d’água $(\mathrm{Pa})$ \\
\hline $\mathrm{e}_{\mathrm{s} \_\min }$ & pressão de saturação calculada a partir da temperatura mínima do ar medida ao nascer do sol \\
\hline$\alpha$ & parâmetro de Priestley-Taylor (adimensional) \\
\hline $\mathrm{r}$ & fator de correção do método da radiação solar (RS). Neste trabalho, específico para as condições de ambiente protegido \\
\hline $\mathrm{R}_{\mathrm{s}}$ & radiação solar global incidente $\left(\mathrm{MJ} \mathrm{d}^{-1} \mathrm{~m}^{-2}\right)$ \\
\hline $\mathrm{k}_{\mathrm{r}}$ & coeficiente de ajuste $\left({ }^{\circ} \mathrm{C}^{-0,5}\right)$ \\
\hline$t_{m}$ & temperatura do ar média para o período considerado $\left({ }^{\circ} \mathrm{C}\right)$ \\
\hline$t_{\min }$ & temperatura do ar mínima diária $\left({ }^{\circ} \mathrm{C}\right)$ \\
\hline $\mathrm{R}_{\mathrm{a}}$ & radiação solar incidente no topo da atmosfera $\left(\mathrm{MJ} \mathrm{d}^{-1} \mathrm{~m}^{-2}\right)$ \\
\hline
\end{tabular}

ou valores extremamente baixos, como os observados em ambiente protegido (Wang et al., 1999; Boulard et al., 2004; Molina-Aiz et al., 2004; Bartzanas et al., 2005). Com a utilização do fator de desacoplamento, é importante observar que a velocidade do ar não é mais necessária para a estimativa do termo $\mathrm{r}_{\mathrm{a}}$.

\section{Penman-Monteith Simplificado (PMAPS)}

Devido à queda acentuada da temperatura do ar no período noturno e às condições microclimáticas específicas de ambientes protegidos, os valores de umidade relativa do ar encontram-se próximos de $100 \%$ ao nascer do sol (Farias et al., 1992; Buriol et al., 2000). Assim, com base nessa característica microclimática, o $\mathrm{DPV}_{\mathrm{ar}}$ ao longo do período diurno pode ser estimado pela diferença entre a pressão de saturação de vapor de água $\left(\mathrm{e}_{\mathrm{s}}\right)$, calculada a partir da temperatura média do ar do período de estimativa da evapotranspiração, e a pressão de saturação $\left(\mathrm{e}_{\mathrm{s} \text { min }}\right)$, calculada a partir da temperatura mínima do ar medida ao nascer do sol. Dessa forma, a equação de Penman-Monteith foi simplificada, conforme apresentado na Tabela 1.

\section{Priestley-Taylor (PT)}

O método de Priestley-Taylor pode ser interpretado como uma simplificação do método originalmente proposto por Penman, em que a evapotranspiração potencial é estimada exclusivamente a partir da evapotranspiração de equilíbrio (McNaughton \& Jarvis, 1983). Nesse caso, um fator de ajuste $\alpha$, universalmente conhecido como parâmetro de Priestley-Taylor, compensa os efeitos do termo aerodinâmico.

\section{Radiação Solar (RS)}

Também conhecido como método da radiação FAO24 , este requer apenas medições da radiação solar $\left(R_{s}\right)$, temperatura do ar no ambiente interno e calibração preliminar para determinação do fator de correção (r), específico do ambiente protegido (Tabela 1).

\section{Radiação Solar Simplificado (RSS)}

Este método é bastante similar ao da radiação solar, no entanto, o termo $\mathrm{R}_{\mathrm{s}}$ é estimado pela equação proposta por Hargreaves \& Samani (1982).

\section{Estimativa do Coeficiente de Cultura}

O coeficiente de cultura $\left(\mathrm{K}_{\mathrm{c}}\right)$ foi determinado para cada um dos cultivares de alface, dividindo-se os valores diários medidos de ET c pelos valores estimados correspondentes da evapotranspiração de referência $\left(\mathrm{ET}_{\mathrm{o}}\right)$. Em seguida, os resultados de $\mathrm{K}_{\mathrm{c}}$ obtidos foram relacionados 
com os graus dia acumulados (GDA) no período póstransplantio, visando à obtenção de um modelo matemático para estimativa posterior desse parâmetro.

Para a estimativa de ET em condições de campo, o método de Penman-Monteith, padronizado como recomendado por Allen et al. (1998), é utilizado como padrão por diversos pesquisadores (Garcia et al., 2004; Katerji \& Rana, 2006; Pereira et al., 2006). Em cultivos protegidos, entretanto, a velocidade do ar está, em geral, abaixo de $0,5 \mathrm{~ms}^{-1}$ na maioria das instalações, mesmo com a utilização de ventilação mecânica (Hanan, 1998). Em razão dessa especificidade microclimática, a resistência aerodinâmica da equação de Penman-Monteith será severamente superestimada se a parametrização proposta por Allen et al. (1998) for implementada, pois esse parâmetro é determinado pela relação $208 / U_{2}$, em que $U_{2}$ é a velocidade do ar medida a dois metros de altura no interior da instalação.

Para corrigir a sensibilidade da resistência aerodinâmica aos baixos valores de velocidade do vento, tipicamente observados em casas de vegetação, foi adotada a parametrização proposta por McNaughton \& Jarvis (1983), em vez da teoria sugerida por Allen et al. (1998).

\section{Análise e Avaliação dos Métodos de Estimativa da Evapotranspiração}

Após a determinação dos parâmetros necessários para implementação dos métodos descritos anteriormente, foi utilizado um conjunto independente de valores medidos das variáveis meteorológicas para estimativa da evapotranspiração da alface (ET $)$ ao longo do ciclo de crescimento. AET foi obtida a partir da multiplicação do coeficiente de cultura $\mathrm{K}_{\mathrm{c}}$ pela evapotranspiração de referência $\left(\mathrm{LE}_{\mathrm{o}}\right.$ ) ou potencial $\left(\mathrm{LE}_{\mathrm{p}}\right)$. Em seguida, os valores de $\mathrm{ET}_{\mathrm{c}}$ estimados foram comparados com os valores medidos. Para teste de desempenho dos métodos de estimativa da evapotranspiração foram calculados o erro médio de estimativa (MBE) e a raiz quadrada do quadrado do erro médio de estimativa (RMSE), conforme sugerido por Jacovides \& Kontoyiannis (1995).

\section{RESULTADOS E DISCUSSÃO}

O modelo sigmoidal com quatro parâmetros ajustouse bem aos valores observados de $\mathrm{K}_{\mathrm{c}}$ para os três cultivares avaliados no período pós-transplantio (Figura 1). Notase que os coeficientes de determinação ajustados foram respectivamente 0,99; 0,98; e 0,98 para os cultivares Grand Rapids, Regina e Great Lakes, mostrando ótimo desempenho deste modelo para estimativa do coeficiente de cultura em sistemas hidropônicos. Informações complementares, como valores numéricos dos parâmetros desse modelo e valores associados do erro-padrão, estão na Tabela 3.
Um modelo similar ao adotado no presente trabalho, denominado equação sigmoidal de Boltzmann, foi empregado por Carmassi et al. (2007) para estimativa do índice de área foliar (IAF) do tomateiro cultivado em sistema hidropônico, tendo como variável independente os grausdia acumulados (GDA) a partir de $400{ }^{\circ} \mathrm{Cd}^{-1}$. Da mesma forma que observado no presente trabalho, os autores encontraram alto coeficiente de determinação $\left(R^{2}=0,994\right)$ entre IAF e GDA, sugerindo também a aplicabilidade deste modelo para estimativa do coeficiente de cultura, uma vez que o $\mathrm{K}_{\mathrm{c}}$ e o IAF estão intrinsecamente relacionados.

Comparando-se os valores obtidos com dados publicados na literatura, específicos para o cultivo da alface em condições de campo, verificam-se discrepâncias enormes no início do período pós-transplantio, mas similaridades após o fechamento do dossel vegetativo. De acordo com Allen et al. (1998), os valores de $\mathrm{K}_{\mathrm{c}}$ recomendados para a alface cultivada em condições de campo são respectivamente 0,$7 ; 1,0$; e 0,9 para os estádios de crescimento inicial, intermediário e final, considerando-se o ciclo de 75 dias, que é muito superior ao da alface produzida em cultivos hidropônicos. Tipicamente, nesse sistema as mudas são produzidas em três semanas e mantidas posteriormente em perfis hidropônicos por um período de quatro semanas, totalizando, assim, um ciclo de aproximadamente 50 dias (Lyra, 2002).

No presente estudo, observa-se que o $\mathrm{K}_{c}$ da alface foi aproximadamente 0,1 no estádio de crescimento inicial (até $150{ }^{\circ} \mathrm{Cd}^{-1}$ ), houve incremento acentuado na fase intermediária, alcançando 0,7 aos $300^{\circ} \mathrm{Cd}^{-1}$, e incremento menor a partir desta data, atingindo no final do ciclo valores em torno de 0,95 para os cultivares Regina e Great Lakes, ambos com formação de cabeça, e 1,1 para o cultivar Grand Rapids, que não apresenta formação de cabeça.

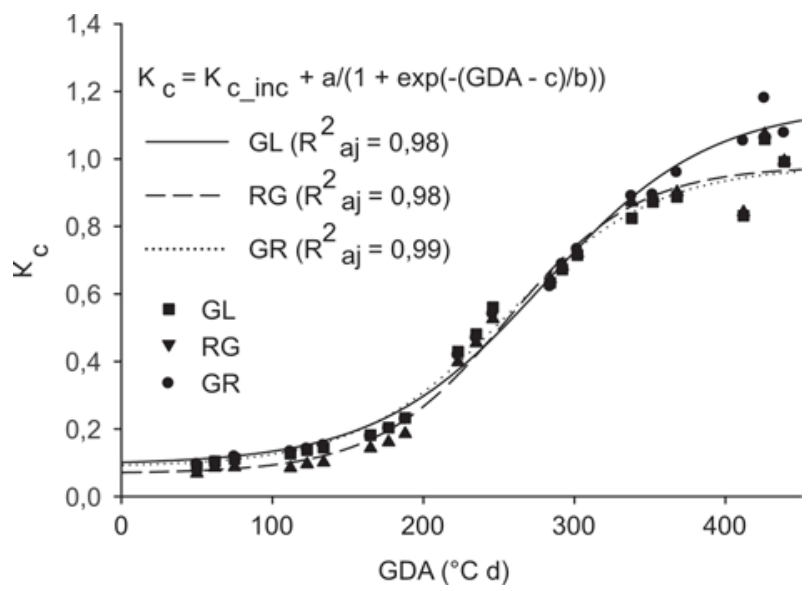

Figura 1. Valores diários estimados (linhas) e medidos (pontos) do coeficiente da cultura $\left(\mathrm{K}_{\mathrm{c}}\right)$ para a alface cultivada em sistema hidropônico em ambiente protegido, tendo como variável independente os graus-dia acumulados após o transplantio (GDA). Os valores apresentados referem-se aos cultivares Grand Rapids (GR), Regina (RG) e Great Lakes (GL). 
Tabela 3. Coeficiente de cultura inicial ( $\mathrm{K}_{\mathrm{c} \text { inc }}$ ), parâmetros do modelo sigmoidal (a, b, c) obtidos a partir de análise de regressão e coeficiente de correlação ajustado para três cultivares de alface produzidos em sistema hidropônico sob ambiente protegido. O erropadrão de estimativa dos parâmetros é apresentado entre parênteses

\begin{tabular}{lccccc}
\hline Cultivar & $\mathbf{K}_{\mathbf{c} \text { inc }}$ & $\mathbf{a}$ & $\mathbf{b}$ & $\mathbf{c}$ & $\mathbf{r}^{2}{ }_{\text {aj }}$ \\
\hline Grand Rapids & $0,0994( \pm 0,0122)$ & $1,065( \pm 0,028)$ & $55,03( \pm 3,15)$ & $279,6( \pm 4,6)$ & 0,99 \\
Regina & $0,0685( \pm 0,0111)$ & $0,910( \pm 0,023)$ & $43,35( \pm 3,36)$ & $255,4( \pm 4,3)$ & 0,98 \\
Great Lakes & $0,0876( \pm 0,0033)$ & $0,891( \pm 0,025)$ & $49,24( \pm 3,78)$ & $254,6( \pm 5,03)$ & 0,98 \\
\hline
\end{tabular}

As diferenças marcantes de $\mathrm{K}_{\mathrm{c}}$ no estádio inicial de crescimento devem ser atribuídas às peculiares desse sistema de cultivo. Em condições de campo, o componente predominante no processo de evapotranspiração no período inicial de crescimento da cultura é a evaporação da água presente no solo (Allen et al., 1998). Em contraste, em sistema hidropônico do tipo NFT, a evaporação tem importância desprezível no processo de evapotranspiração, uma vez que esse ocorre apenas através dos orifícios do perfil hidropônico. Paralelamente, a transpiração da cultura é pouco expressiva nesse estágio de crescimento, tendo em vista que o índice de área foliar da cultura é muito baixo.

Na Figura 2, observa-se a variação da irradiância solar global diária $\left(\mathrm{R}_{\mathrm{g}}\right)$ e dos valores médios diurnos (Figuras 2A e 2B) e noturnos (Figuras 2C e 2D) do déficit de pres-
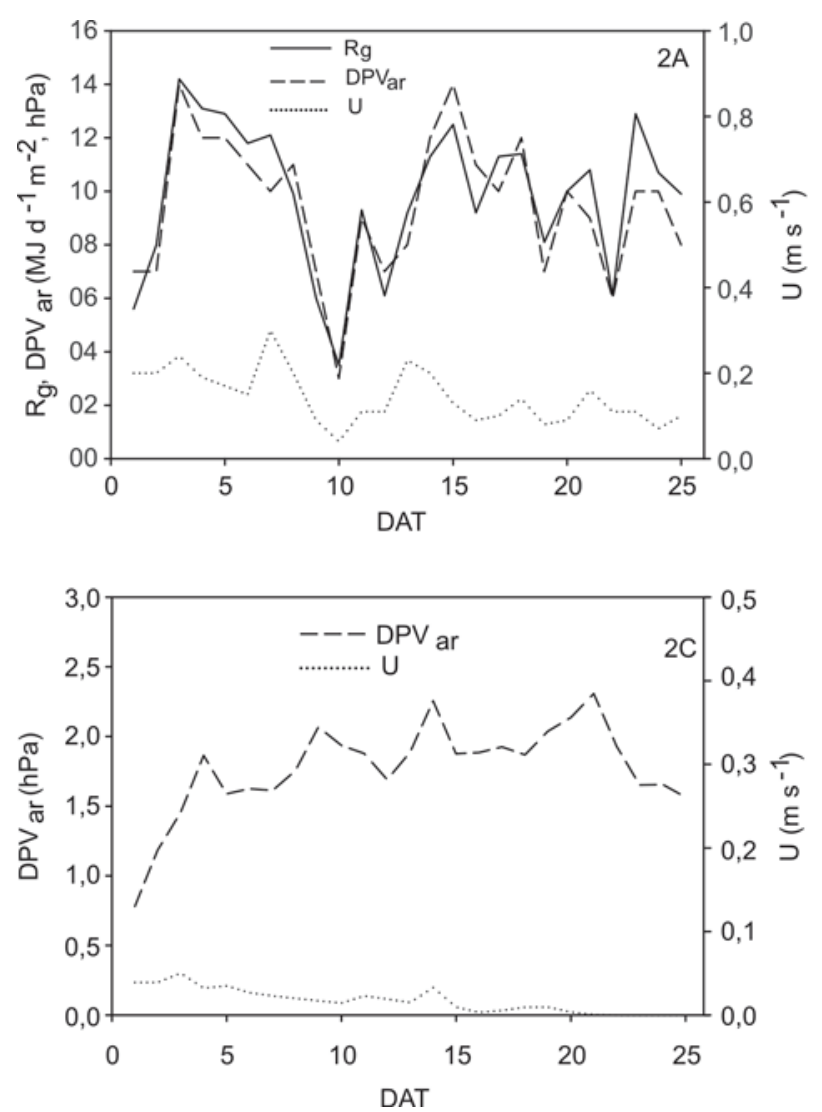

são de saturação de vapor de água no ar (DPV $\left.{ }_{a r}\right)$, velocidade do ar (U), temperatura do ar (t) e umidade relativa do ar (UR) ao longo do ciclo de crescimento da alface hidropônica, expresso em dias após o transplantio (DAT). Os valores exibidos nessa figura foram empregados nos diversos métodos de estimativa de ET $\mathrm{T}_{c}$ para os três cultivares de alface.

Os valores de $\mathrm{R}_{\mathrm{g}}$ oscilaram de 3 a $14 \mathrm{MJ} \mathrm{d}^{-1} \mathrm{~m}^{-2}$ no período compreendido entre a data de transplantio e 25 DAT, em decorrência da aproximação de duas frentes frias e de condições parcialmente nubladas, características do clima da localidade em estudo. Assim, essas duas condições ditaram o comportamento da temperatura, umidade relativa e do déficit de pressão de vapor de água no ar. Os valores diurnos de t, UR e DPV ${ }_{\text {ar }}$ estiveram compreendidos entre 19 e $25^{\circ} \mathrm{C}$, 50 e $85 \%$ e 4 e $14 \mathrm{hPa}$, respectivamente.
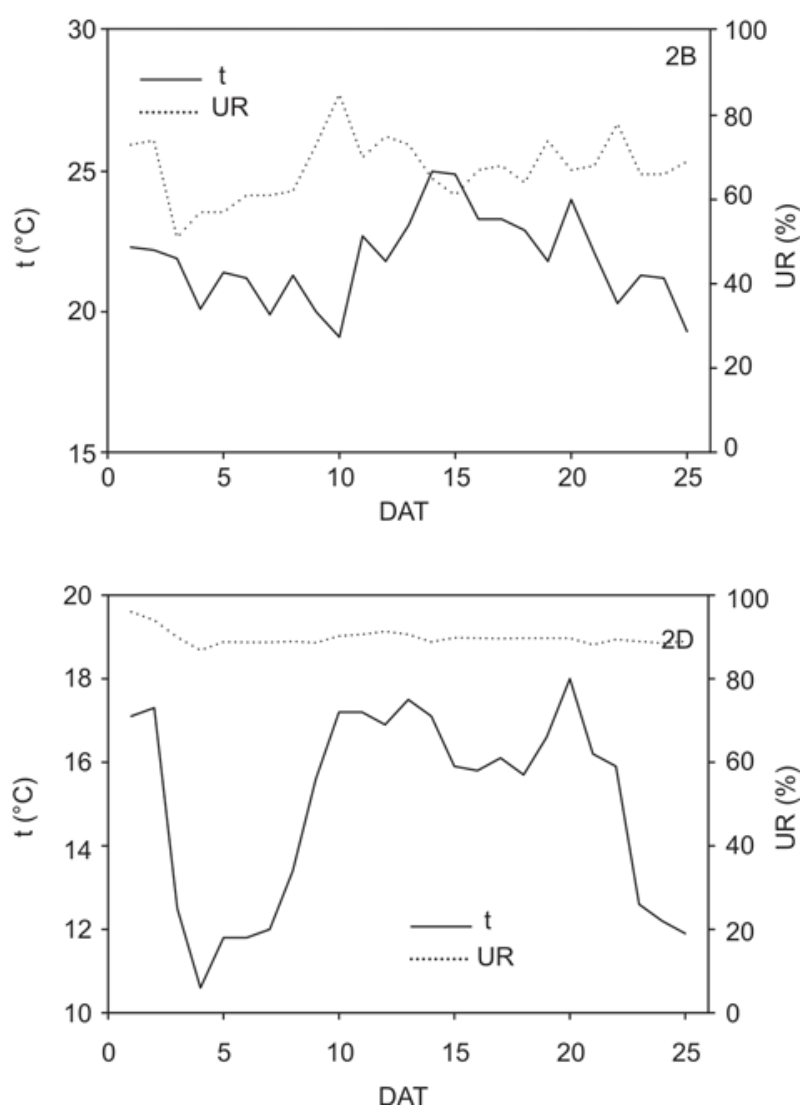

Figura 2. Variação da irradiância solar global diária $\left(R_{g}\right)$ e dos valores médios diurnos (Figuras 2A e 2B) e noturnos (Figuras 2C e 2D) do déficit de pressão de saturação de vapor d'água no ar $\left(\mathrm{DPV}_{\mathrm{ar}}\right)$, velocidade do ar $(\mathrm{U})$, temperatura do ar $(\mathrm{t})$ e umidade relativa do ar (UR) ao longo do ciclo de crescimento da alface hidropônica, expresso em dias após o transplantio (DAT). 
No período noturno, a UR apresentou pequenas variações, em torno de $90 \%$. Em contraste, a temperatura do ar foi severamente afetada pela chegada das frentes frias anteriormente mencionadas, sendo uma 3 DAT e outra 22 DAT. Apesar da variação marcante da temperatura do ar à noite, o $\mathrm{DPV}_{\mathrm{ar}}$ variou somente entre 0,9 e 2,3 hPa, como resultado da alta umidade relativa.

Devido à utilização de telas plásticas nas laterais da instalação para evitar a presença de insetos no ambiente de cultivo, a velocidade do ar esteve sempre abaixo de 0,3 $\mathrm{m} \mathrm{s}^{-1}$ no período diurno e inferior a $0,05 \mathrm{~m} \mathrm{~s}^{-1}$ à noite. Portanto, nem mesmo a instabilidade atmosférica, oriunda da aproximação das frentes frias durante o período experimental, foi capaz de intensificar o movimento do ar no interior da casa de vegetação. Resultados semelhantes, relacionados a baixos valores de velocidade do ar em ambientes protegidos, também foram relatados por Wang et al. (1999), Boulard et al. (2004), Molina-Aiz et al. (2004) e Bartzanas et al. (2005).

Na Tabela 4 encontram-se os valores do erro médio de estimativa (MBE) e da raiz quadrada do quadrado do erro médio de estimativa (RMSE), referentes ao desempenho estatístico dos diferentes métodos utilizados para estimativa diária de evapotranspiração dos cultivares de alface Grand Rapids, Regina e Great Lakes. Os resultados foram organizados com o intuito de mostrar a variação de desempenho dos métodos ao longo do ciclo da cultura, que foi dividido em cinco campanhas de medição a partir do transplantio. Cada etapa de mensuração de $\mathrm{ET}_{\mathrm{c}}$ compreendeu um período

Tabela 4. Desempenho estatístico dos métodos PMF, PMAP, PMAPS, PT, RS e RSS para estimativa da evapotranspiração diária dos cultivares de alface Grand Rapids, Regina e Great Lakes em sistema hidropônico em ambiente protegido. O período experimental compreendeu cinco etapas de medição, cada uma com duração de três dias, que foram conduzidas após o transplantio

\begin{tabular}{|c|c|c|c|c|c|c|c|}
\hline \multirow[b]{2}{*}{ Etapas } & \multirow[b]{2}{*}{ Método } & \multicolumn{2}{|c|}{ Grand Rapids } & \multicolumn{2}{|c|}{ Regina } & \multicolumn{2}{|c|}{ Great Lakes } \\
\hline & & $\begin{array}{c}\text { RMSE } \\
\left(\mathrm{kg} \mathrm{d}^{-1} \mathrm{~m}^{-2}\right)\end{array}$ & $\begin{array}{c}\mathrm{MBE} \\
\left(\mathrm{kg} \mathrm{d}^{-1} \mathrm{~m}^{-2}\right)\end{array}$ & $\begin{array}{c}\text { RMSE } \\
\left(\mathrm{kg} \mathrm{d}^{-1} \mathrm{~m}^{-2}\right)\end{array}$ & $\begin{array}{c}\mathrm{MBE} \\
\left(\mathrm{kg} \mathrm{d}^{-1} \mathrm{~m}^{-2}\right)\end{array}$ & $\begin{array}{c}\text { RMSE } \\
\left(\mathrm{kg} \mathrm{d}^{-1} \mathrm{~m}^{-2}\right)\end{array}$ & $\begin{array}{c}\mathrm{MBE} \\
\left(\mathrm{kg} \mathrm{d}^{-1} \mathrm{~m}^{-2}\right)\end{array}$ \\
\hline \multirow{6}{*}{$1^{\circ}$} & PMF & 0,07 & $-0,07$ & 0,08 & $-0,07$ & 0,09 & $-0,09$ \\
\hline & PMAP & 0,02 & 0,01 & 0,04 & $-0,03$ & 0,03 & $-0,02$ \\
\hline & PMAPS & 0,05 & 0,03 & 0,05 & $-0,02$ & 0,04 & $-0,01$ \\
\hline & $\mathrm{PT}$ & 0,02 & $-0,01$ & 0,03 & $-0,02$ & 0,05 & $-0,04$ \\
\hline & $\mathrm{RS}$ & 0,05 & 0,04 & 0,07 & $-0,06$ & 0,02 & 0,01 \\
\hline & RSS & 0,26 & 0,24 & 0,11 & 0,07 & 0,19 & 0,18 \\
\hline \multirow{6}{*}{$2^{o}$} & PMF & 0,07 & $-0,07$ & 0,05 & $-0,04$ & 0,09 & $-0,08$ \\
\hline & PMAP & 0,02 & 0,01 & 0,01 & 0,01 & 0,03 & $-0,01$ \\
\hline & PMAPS & 0,05 & 0,04 & 0,02 & 0,02 & 0,01 & 0,01 \\
\hline & $\mathrm{PT}$ & 0,04 & $-0,03$ & 0,01 & $-0,01$ & 0,05 & $-0,05$ \\
\hline & RS & 0,04 & 0,02 & 0,04 & $-0,01$ & 0,04 & $-0,01$ \\
\hline & RSS & 0,46 & 0,45 & 0,27 & 0,27 & 0,37 & 0,36 \\
\hline \multirow{6}{*}{$3^{\circ}$} & PMF & 0,11 & $-0,10$ & 0,09 & 0,07 & 0,09 & $-0,09$ \\
\hline & PMAP & 0,15 & 0,13 & 0,27 & 0,24 & 0,14 & 0,12 \\
\hline & PMAPS & 0,25 & 0,21 & 0,35 & 0,32 & 0,23 & 0,20 \\
\hline & $\mathrm{PT}$ & 0,19 & 0,17 & 0,36 & 0,33 & 0,17 & 0,16 \\
\hline & RS & 0,01 & $-0,01$ & 0,04 & 0,03 & 0,02 & 0,01 \\
\hline & RSS & 0,79 & 0,77 & 0,70 & 0,67 & 0,71 & 0,69 \\
\hline \multirow{6}{*}{$4^{\circ}$} & PMF & 0,22 & 0,08 & 0,11 & 0,05 & 0,18 & $-0,15$ \\
\hline & PMAP & 0,51 & 0,44 & 0,38 & 0,35 & 0,20 & 0,16 \\
\hline & PMAPS & 0,59 & 0,54 & 0,44 & 0,44 & 0,27 & 0,25 \\
\hline & $\mathrm{PT}$ & 0,50 & 0,46 & 0,46 & 0,43 & 0,23 & 0,20 \\
\hline & RS & 0,50 & 0,38 & 0,18 & 0,17 & 0,15 & 0,07 \\
\hline & RSS & 1,75 & 1,69 & 1,40 & 1,37 & 1,30 & 1,25 \\
\hline \multirow{6}{*}{$5^{\circ}$} & $\mathrm{PMF}$ & 0,15 & 0,05 & 0,14 & $-0,02$ & 0,14 & $-0,10$ \\
\hline & PMAP & 0,37 & 0,33 & 0,27 & 0,23 & 0,19 & 0,16 \\
\hline & PMAPS & 0,68 & 0,65 & 0,54 & 0,51 & 0,46 & 0,44 \\
\hline & $\mathrm{PT}$ & 0,67 & 0,64 & 0,54 & 0,50 & 0,41 & 0,39 \\
\hline & RS & 0,23 & 0,14 & 0,19 & 0,06 & 0,14 & 0,08 \\
\hline & RSS & 1,88 & 1,86 & 1,58 & 1,57 & 1,58 & 1,57 \\
\hline
\end{tabular}

Rev. Ceres, Viçosa, v. 57, n.2, p. 224-233, mar/abr, 2010 
contínuo com duração de três dias, que foi interrompida por dois dias, estabelecendo, a partir do seu término, o início da próxima etapa de medição. A primeira etapa teve início no período compreendido entre o $3^{\circ}$ e $5^{\circ}$ dia após o tranplantio, quando o índice de área foliar (IAF) médio para os três cultivares e repetições alcançou 0,12. Na segunda, terceira, quarta e quinta etapas, o IAF médio foi 0,$26 ; 0,62 ; 1,64$; e 3,14, respectivamente.
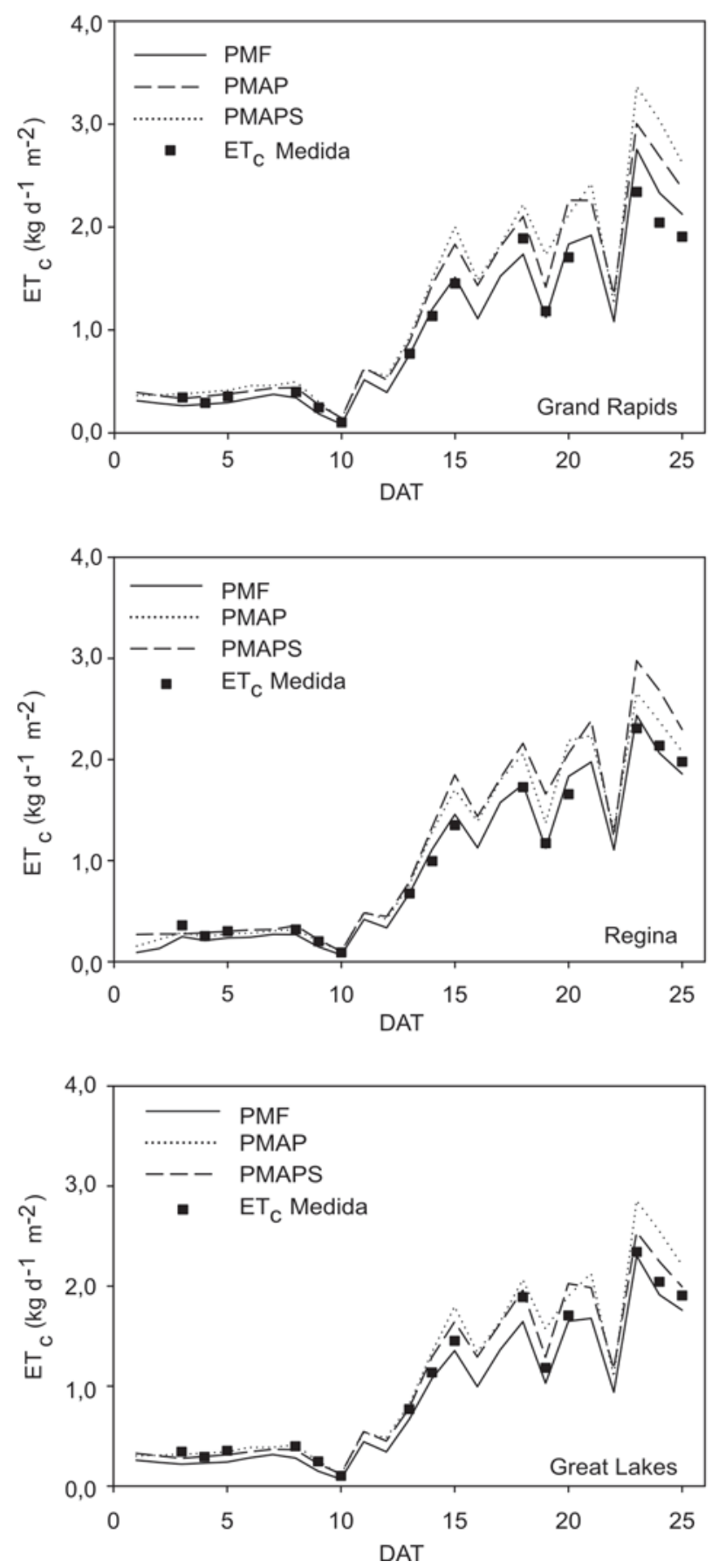

Com exceção do método RSS, que apresentou erros bem acima dos demais, observa-se que o erro de precisão (RMSE) foi inferior a $0,10 \mathrm{~kg} \mathrm{~d}^{-1} \mathrm{~m}^{-2}$ nas duas primeiras etapas de medição. No entanto, com o crescimento da cultura, o RMSE aumentou a partir dessa etapa, acompanhando a expansão foliar e, consequentemente, a evapotranspiração. No final do período experimental, o RMSE alcançou $0,68 \mathrm{~kg} \mathrm{~d}^{-1} \mathrm{~m}^{-2}$ nas estimativas realizadas para o cultivar Grand Rapids, por meio do método PMAPS.
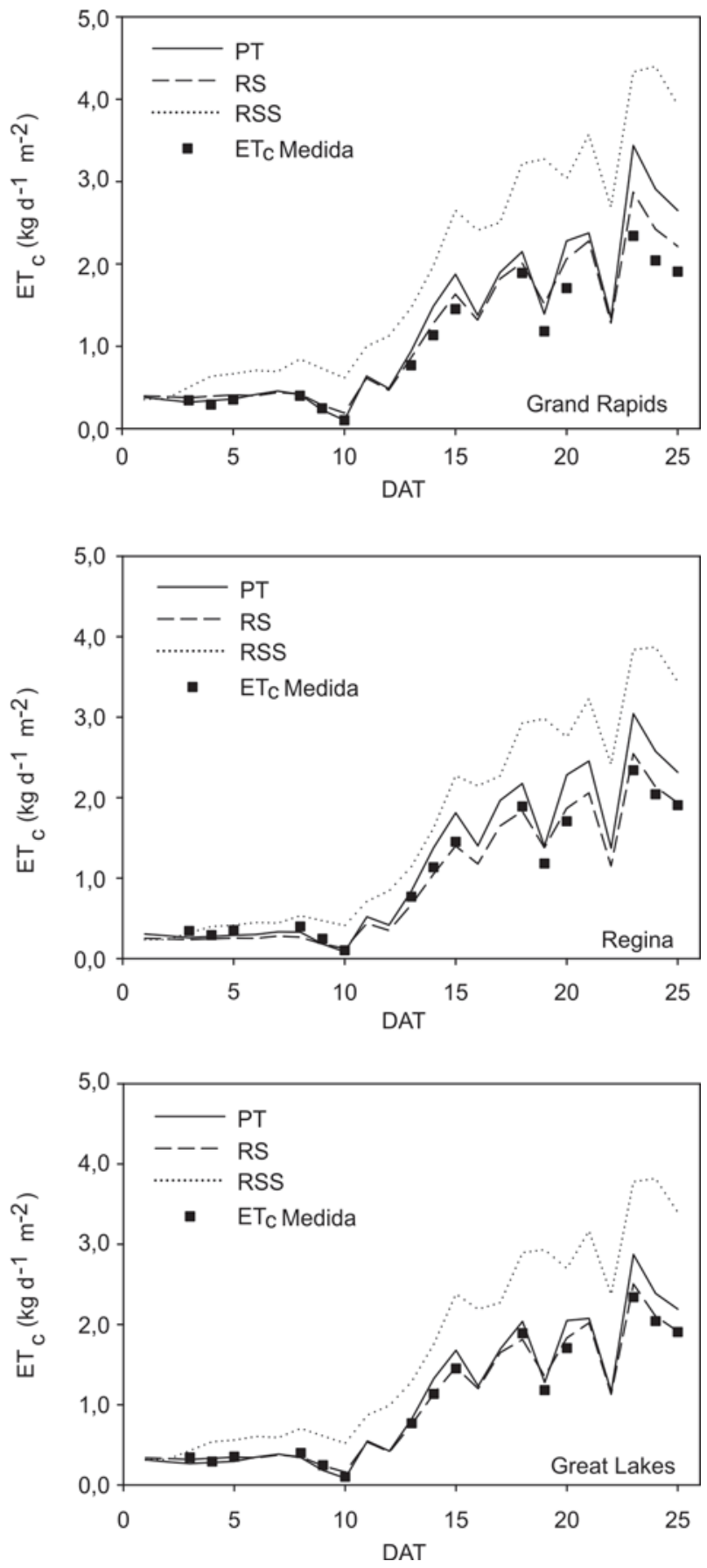

Figura 3. Variação dos valores medidos e estimados da evapotranspiração (ET) de três cultivares de alface ao longo do ciclo de crescimento em sistema hidropônico sob ambiente protegido. As estimativas de $\mathrm{ET}_{\mathrm{c}}$ foram realizadas por meio dos métodos PMF, PMAP e PMAPS (lado esquerdo) e PT, RS e RSS (lado direito). 
No entanto, o índice RMSE foi bem maior quando o método RSS foi utilizado, chegando a $1,88 \mathrm{~kg} \mathrm{~d}^{-1} \mathrm{~m}^{-2}$ para o cultivar Grand Rapids. Comportamento semelhante foi constatado para a avaliação do índice de exatidão que, em termos absolutos, foi inferior a $0,10 \mathrm{~kg} \mathrm{~d}^{-1} \mathrm{~m}^{-2}$ no início do ciclo da cultura e alcançou $1,86 \mathrm{~kg} \mathrm{~d}^{-1} \mathrm{~m}^{-2}$ para o mesmo cultivar na última campanha de medição.

A partir da análise do sinal do índice MBE, constatouse também que, de maneira geral, os métodos PMF, PMAP e RS apresentaram melhor desempenho estatístico em relação aos métodos PMAPS, PT e RSS, independentemente do cultivar avaliado. No entanto, o método PMF, padronizado como sugerido por Allen et al. (1998), subestimou sistematicamente a $\mathrm{ET}_{\mathrm{c}}$ dos três cultivares de alface nas duas primeiras campanhas de medição.

A padronização proposta por McNaughton \& Jarvis (1983) possibilitou estimativas diárias mais exatas da evapotranspiração, subestimando ou superestimando a $\mathrm{ET}_{\mathrm{c}}$ dentro da faixa de $-0,03 \mathrm{a}+0,04 \mathrm{~kg} \mathrm{~d}^{-1} \mathrm{~m}^{-2}$. Entretanto, a simplificação do método PMAP, o qual assume que a pressão média diária de vapor de água no ar é igual à pressão de saturação ao nascer do sol, tendeu a superestimar levemente $\mathrm{ET}_{\mathrm{c}}$ a partir da terceira etapa de medição (IAF médio de 1,64). Por outro lado, deve-se ressaltar que esse método não necessita de medições de umidade relativa do ar no ambiente de cultivo e pode ser uma alternativa para implementação de sistemas automáticos de baixo custo para manejo de irrigação em ambientes protegidos.

Os métodos empíricos PT, RS e RSS apresentaram resultados similares ou discrepantes, dependendo da etapa de medição da ET . Na fase inicial de crescimento da cultura, o método PT também subestimou ligeiramente a ET, e a partir desse período tendeu a superestimá-la. Por outro lado, os métodos baseados na radiação solar, de forma geral, tenderam a superestimá-la, especialmente no final do ciclo da cultura. O método RSS, que requer apenas dados de temperatura do ar e adota a simplificação proposta por Hargreaves \& Samani (1982) para estimativa da radiação solar global, superestimou a ET sistematicamente ao longo do ciclo da cultura. Neste trabalho, os valores dos parâmetros empíricos $\alpha$, r e $\mathrm{k}_{\mathrm{r}}$, pertinentes aos métodos PT, RS e RSS, foram 1,26 ( 0,03), 0,73 ( $\pm 0,03)$ e 0,18 ( \pm 0,01), respectivamente.

Com o objetivo de complementar os dados apresentados na Tabela 4, a Figura 3 foi elaborada para ilustrar a variação dos valores medidos e estimados da evapotranspiração $\left(\mathrm{ET}_{\mathrm{c}}\right)$ dos três cultivares de alface no período pós-transplantio. Nessa figura, observa-se que todos os métodos acompanharam as variações das condições meteorológicas no ambiente de cultivo. No início do ciclo de crescimento, a ET da $_{\text {d }}$ alface foi de aproximadamente $0,2 \mathrm{~kg} \mathrm{~d}^{-1} \mathrm{~m}^{-2}$, e aumentou aproximadamente 10 vezes até alcançar $2,0 \mathrm{~kg} \mathrm{~d}^{-1} \mathrm{~m}^{-2}$ no final do ciclo da cultura. As oscilações da $\mathrm{ET}_{\mathrm{c}}$ são decorrentes das variações de nebulosidade e presença de duas frentes frias, conforme descrito anteriormente (Figura 2). Nota-se, como já ressaltado, o afastamento sistemático da linha de $E_{\text {c }}$ estimada pelo método RSS em relação aos valores medidos de evapotranspiração dos três cultivares de alface avaliados.

\section{CONCLUSÕES}

Em cultivos hidropônicos em ambiente protegido, o modelo sigmoidal com quatro parâmetros, tendo como variável independente os graus-dia acumulados após o transplantio, apresenta excelentes ajustes aos valores observados do coeficiente de cultura para os cultivares de alface Grand Rapids, Regina e Great Lakes.

De forma geral, os métodos PMF, PMAP e RS apresentam melhor desempenho estatístico em relação aos PMAPS, PT e RSS, independentemente do cultivar avaliado. Entretanto, embora o método PMAPS tenha apresentado leve tendência a superestimar a $\mathrm{ET}_{\mathrm{c}}$ após o fechamento do dossel vegetativo, este método não necessita de medições de velocidade do ar e de umidade relativa no ambiente de cultivo. Dessa forma, constitui-se em alternativa para implementação de sistemas automáticos de baixo custo para manejo de irrigação em ambientes protegidos.

\section{REFERÊNCIAS}

Allen RG, Pereira LS, Raes D \& Smith M (1998) Crop evapotranspiration: guidelines for computing crop water requirements. Roma, FAO, Irrigation and Drainage Paper 56. 300p.

Amayreh J \& Al-Abed N (2005) Developing crop coefficients for field-grown tomato (Lycopersicon esculentum Mill.) under drip irrigation with black plastic mulch. Agricultural Water Management, 73:247-254.

Bartzanas T, Tchamitchian M \& Kittas C (2005) Influence of the heating method on greenhouse microclimate and energy consumption. Biosystems Engineering, 91:487-499.

Boulard T, Fatnassi H, Roy JC, Lagier J, Fargues J, Smits N, Rougier M \& Jeannequin B (2004) Effect of greenhouse ventilation on humidity of inside air and in leaf boundary-layer. Agricultural and Forest Meteorology, 125:225-239.

Buriol GA, Righi EZ, Schneider FM, Streck NA, Heldwein AB \& Estefanel V (2000) Modificação da umidade relativa do ar pelo uso e manejo da estuda plástica. Revista Brasileira de Agrometeorologia, 8:11-18.

Carmassi G, Incrocci L, Maggini R, Malorgio F, Tognoni F \& Pardossi A (2007) An aggregated model for water requirements of greenhouse tomato grown in closed rockwool culture with saline water. Agricultural Water Management, 88:73-82.

Doorenbos J \& Pruitt W (1977) Guidelines for predicting crop water requirements. Roma, FAO: Irrigation and Drainage Paper 24. $144 p$.

Farias JRB, Bergamamaschi H, Martins SR, Berlato MA \& Oliveira ACB (1992) Alterações na temperatura e umidade relativa do ar provocadas pelo uso de estufa plástica. Revista Brasileira de Agrometeorologia, 1:51-62. 
Garcia M, Raes D, Allen R \& Herbas C (2004) Dynamics of reference evapotranspiration in the Bolivian highlands (Altiplano). Agricultural and Forest Meteorology, 125:67-82.

Hanan JJ (1998) Greenhouses - Advanced technology for protected horticulture. Boca Raton, CRC Press LLC. 684p.

Hargreaves GH \& Samani ZA (1982) Estimating potential evapotranspiration. Journal of Irrigation and Drainage Engineering, 108:225-230.

Jacovides CP \& Kontoyiannis H (1995) Statistical procedures for the evaluation of evapotranspiration computing models. Agricultural Water Management, 27:365-371.

Jayanthi H, Naele CMU \& Wright JL (2007) Development and validation of canopy reflectance-based crop coefficient for potato. Agricultural Water Management, 88:235-246.

Katerji N \& Rana G (2006) Modelling evapotranspiration of six irrigated crops under Mediterranean climate conditions. Agricultural and Forest Meteorology, 138:142-155.

Lyra GB (2002) Estimativa da evapotranspiração e análise de crescimento para alface (Lactuca sativa L.) cultivada em sistema hidropônico em condições de casa-de-vegetação. Dissertação de Mestrado. Universidade Federal de Viçosa, Viçosa, $91 \mathrm{p}$.
McNaughton KG \& Jarvis PG (1983) Predicting effects of vegetation changes on transpiration and evaporation. In: Kozlowski TT (Ed.), Water deficits and plant growth, vol. VII. New York, Academic Press. p.1-47.

Molina-Aiz FD, Valera DL \& Álvarez AJ (2004) Measurement and simulation of climate inside Almería-type greenhouses using computational fluid dynamics. Agricultural and Forest Meteorology, 125:33-51.

Oliveira AS de, Martin EC, Slack DC, Pegelow EJ \& Folta AD (2005) Water use and crop coefficient of subsurface drip-irrigated lettuce in Central Arizona. Revista Brasileira de Engenharia Agrícola e Ambiental, 9:37-44.

Orgaz F, Fernández MD, Bonachela S, Gallardo M \& Fereres E (2005) Evapotranspiration of horticultural crops in an unheated plastic greenhouse. Agricultural Water Management, 72:81-96.

Pereira AR, Green S \& Villa Nova NA (2006) Penman-Monteith reference evapotranspiration adapted to estimate irrigated tree transpiration. Agricultural Water Management, 83:153-161.

Wang S, Boulard T \& Haxaire R (1999) Air speed profiles in a naturally ventilated greenhouse with a tomato crop. Agricultural and Forest Meteorology, 96:181-188.

Zolnier S, Lyra GB \& Gates RS (2004) Evapotranspiration estimates for greenhouse lettuce using an intermittent nutrient film technique. Transactions of the ASAE, 47:271-282. 